1

Noname manuscript No.

(will be inserted by the editor)

\title{
Consistency problems in the improvement of the IAU precession-nutation theories: effects of the dynamical ellipticity differences
}

\author{
Alberto Escapa · José M. Ferrándiz • \\ Tomás Baenas • Juan Getino • Juan F. \\ Navarro • Santiago Belda-Palazón \\ Received: date / Accepted: date \\ Alberto Escapa \\ Department of Applied Mathematics, University of Alicante, P.O. Box 99, E-03080 Alicante, \\ Spain. E-mail: alberto.escapa@ua.es \\ José M. Ferrándiz \\ Department of Applied Mathematics, University of Alicante, P.O. Box 99, E-03080 Alicante, \\ Spain. E-mail: jm.ferrandiz@ua.es \\ Tomás Baenas \\ Department of Applied Mathematics, University of Alicante, P.O. Box 99, E-03080 Alicante, \\ Spain. E-mail: tomas.baenas@ua.es \\ Juan Getino \\ Department of Applied Mathematics, University of Valladolid, E-47011 Valladolid, Spain. E- \\ mail: getino@maf.uva.es \\ Juan F. Navarro \\ Department of Applied Mathematics, University of Alicante, P.O. Box 99, E-03080 Alicante, \\ Spain. E-mail: jf.navarro@ua.es \\ Santiago Belda-Palazón
}


Abstract The complexity of the modelling of the rotational motion of the Earth in space has produced that no single theory has been adopted to describe it in full. Hence, it is customary using at least a theory for precession and another one for nutation. The classic approach proceeds by deriving some of the fundamental parameters from the precession theory, like, e.g. the dynamical ellipticity $H_{d}$, and then using those values in the nutation theory. The former IAU 1976 precession and IAU 1980 nutation theories followed that scheme. Along with the improvement of the accuracy of the determination of EOP (Earth Orientation Parameters), IAU 1980 was superseded by IAU2000, based on the application of the MHB2000 transfer function to the previous rigid earth analytical theory REN2000. The latter was derived while the precession model IAU 1976 was still in force, therefore it used the corresponding values for some of the fundamental parameters, as the precession rate, associated to the dynamical ellipticity. The new precession model P03 was adopted as IAU 2006. That change introduced some inconsistency since P03 used different values for some of the fundamental parameters that MHB2000 inherited from REN2000. Besides, the derivation of the basic Earth parameters of MHB2000 itself comprised a fitted variation of the dynamical ellipticity adopted in the background rigid theory. Due to the strict requirements of accuracy of the present and coming times, the magnitude of the inconsistencies originated by this two-fold approach is no longer negligible as earlier, hence the need of discussing the effects of considering slightly different values for $H_{d}$ in precession and nutation theories.

Department of Applied Mathematics, University of Alicante, P.O. Box 99, E-03080 Alicante, Spain. E-mail: santiago.belda@ua.es 
Keywords Earth Rotation - Precession and nutation - Reference systems -

Celestial Mechanics

\section{Introduction}

Earth rotation is presently a very active field of research, in which mathematical and physical methods concur to satisfy the very stringent demands of accuracy resulting from a broad set of applications to various fields. A comprehensive description of the geodetic problems and the expected societal benefits which led to the establishment of the requirements of such high accuracy can be seen, e.g., in Plag and Pearlman's book (2009) on GGOS, the Global Geodetic Observing System promoted by the International Association of Geodesy (IAG).

To respond to the scientific challenges associated with the rapidly increasing requirements associated to geodetic observations (Plag et al. 2009), GGOS demands improved consistency to all IAG products at the Horizon 2020, as well as and accuracy of the order of $1 \mathrm{~mm}$ to the frames of reference, besides a stability in time of $0.1 \mathrm{~mm} / \mathrm{y}$. The former accuracy in position, measured on the Earth surface, corresponds roughly to an angle of $30 \mu$ as from the Earth's centre. The realizations of the Terrestrial Reference Frames (TRF) depends on complex processes and are highly correlated with the determination of the Earth Orientation Parameters (EOP), which provide the time dependent rotation relating the Terrestrial to the Celestial Reference Frames (CRF) - see, e.g. Heinkelmann et al. (2015a, 2015b).

From the observational side, the accuracy and performance of the major Space Geodesy techniques contributing to the EOP determination is noticeably increas- 
ing. A good example is provided by the deployment of a new generation of VLBI

(Very Long Baseline Interferometry) stations compliant with the GGOS/IAG 2010 specifications, the so-called VGOS. It can be expected that more accurate EOP time series will be produced in a few years, even at a sub-daily rate, following the experience of continuous VLBI campaigns (Nilsson et al. 2010). That would be useful to overcome deficiencies in the models used to describe diurnal and sub-diurnal variations of EOPs (Böhm et al. 2012).

In this context, the International Astronomical Union (IAU) and the IAG set up a new Joint Working Group on Theory of Earth Rotation (JWG_ThER). It was aimed at promoting the development of theories of Earth rotation that are fully consistent and that agree with observations and provide predictions of EOP with the accuracy required to meet the needs of the near future; its operation started in 2013. Consistency at the targeted level of accuracy is one of the main topics of interest since the beginning of the activities of that JWG.

Within this framework we examine the effect stemmed by the simultaneous assignment of more than one value to a same parameter in different components of the precession and nutation theories. Since the modeling of the Earth rotation is extremely complex, its motion is usually described considering one theory for its long-term behavior, precession, and other for the short-term one, nutation. This two-fold approach has the consequence that the parameters entering in the description are not given exactly the same values in precession and nutation theories, as it should be obviously. This lack of consistency might originate numerical differences that would be incompatible with nowadays accuracy requirements. The situation is even more complicated, because the current nutation model, IAU2000, is, as well, made up of two different theories, namely., the rigid Earth theory 
REN2000 (Souchay et al. 1999) and the non-rigid transfer function of MHB2000

(Mathews et al. 2002) that is applied to REN2000 terms.

In particular, we focus on the slightly differences considered in precession and nutation models for the numerical values of the dynamical ellipticity ${ }^{1} H_{d}$, with $H_{d}=(C-\bar{A}) / C$, being $C$ the polar and $\bar{A}$ the mean equatorial moments of inertia of the Earth, since it is a key parameter in the determination and understanding of the rotation of the Earth.

Basically, the main differences arise because the dynamical ellipticity considered in the current precession model, IAU 2006 (Hilton et al. 2006), introduced a time dependent part, due to the $J_{2}$ time rate, not considered in IAU2000 nutation model. In turn, second order effects coming from the nutation-nutation coupling, in the sense of perturbation theories, induce a net contribution to the value of precession, hence producing a change of $H_{d}$ not taken into account in IAU 2006 precession model. Next, we discuss the scope of those inconsistencies and estimate their numerical influence in the precession and nutation of the Earth.

The paper is structured as follows. In Section 2 we sketch the main features of the official standards of precession and nutation adopted by IAU and in force nowadays. We show the potential sources of inconsistencies motivated by the coexistence of three different dynamical models that serve as the basis of the nutation and precessional ones. The adjustments of the nutational part to the precessional one, to achieve higher levels of consistency, are examined in Section 3, whereas

\footnotetext{
1 Some authors prefer the denomination of dynamical flattening (e.g., Huang et al. 2014). Here, we follow the terminology employed in Kinoshita (1977), Moritz and Mueller (1987) or Williams (1994), among others. Different names such as mechanical ellipticity can also be found (e.g., Heisanken and Moritz 1967).
} 
those induced by improvements in the mathematical methods of solution or some selected, previously unaccounted, geophysical effects on the precession and nutations are presented in Section 4. Finally, we draw some general conclusions about their implications on the current requirements of accuracy of precession-nutation theories.

\section{Background: current precession and nutation models}

The General Assemblies (GA) of the International Astronomical Union (IAU) held in 2000 and 2006 approved transcendental Resolutions relative to the precession and nutation models that are widely used in Astronomy and Space or Earth Observation Sciences.

Specifically, the XXIVth IAU GA (2000) Resolution B1.6 endorsed the IAU2000A precession-nutation model. IAU2000A was intended for users needing accuracy at the 0.2 mas (mili-arcsecond) level. Its nutational component consists of the convolution of the MHB2000 transfer function with a pre-existing rigid Earth model, REN2000. Its precessional component is basically that of IAU 1976 (Lieske et al. 1977), updated with corrections to the precession rates and offsets. The development of new expressions for precession consistent with IAU2000A was then encouraged.

Six years later, the XXVIth IAU GA (2006) Resolution B1 adopted the IAU 2006 precession model. The precession component of the IAU2000A precessionnutation model was replaced by the P03 precession theory (Capitaine et al. 2003), although the model developed by Fukushima (2003) also provided a very complete description of the precessional motion, including a new parameterization of the 
angles that is one of the alternatives recommended in the IERS Conventions 2010.

P03 model gave new values to the precession rate in longitude and the obliquity of the equator, more accurate than those of Lieske et al. (1977), including also a conventional $J_{2}$ time rate and polynomial expansions of higher degree for the fundamental angles, e.g.,

$$
\begin{aligned}
\dot{J}_{2}= & -3 \times 10^{-9} \mathrm{cy}^{-1} \\
\psi_{A}= & 5038^{\prime \prime} .481507 t-1^{\prime \prime} .0790069 t^{2}-0^{\prime \prime} .00114045 t^{3}+0^{\prime \prime} .000132851 t^{4}-0^{\prime \prime} .000000951 t^{5} \\
\epsilon_{A}= & 84381^{\prime \prime} .406-46^{\prime \prime} .836769 t-0^{\prime \prime} .0001831 t^{2}+0^{\prime \prime} .00200340 t^{3} \\
& -0^{\prime \prime} .000000576 t^{4}-0^{\prime \prime} .0000000434 t^{5}
\end{aligned}
$$

Here $\psi_{A}$ and $\epsilon_{A}$ are the angles providing the precession of the equator in longitude and obliquity (Capitaine et al. 2003), respectively, and $t$ is measured in Julian centuries since JD2000.0.

At the highest levels of precision, the replacement of the precessional part of IAU2000 by P03 is not trivial: the three main components of the IAU2000 and IAU 2006 precession-nutation theories (REN2000, MHB2000, P03) assign slightly different values to several astronomical and geophysical parameters with identical definition, due in part to their updating. That source of inconsistency was already pointed in a first analysis by Capitaine et al. (2005).

At this point, it is convenient to recall some basic features of the implied theories. The rigid Earth theory REN2000 is mainly analytical and provides expressions for both precession and nutation, unlike the non-rigid theories based on the transfer function approach. It uses the Hamiltonian formalism, following the method first developed by Kinoshita (1977), both regarding the used canonical variables and the derivation of the solution by means of perturbations methods 
(in particular the Lie-Hori canonical one, Hori 1966, Kinoshita 1977); the tide generating potential (first order gravitational disturbing potential) is expanded in terms of the canonical Andoyer's variables and the five classic Delaunay's angles appearing in the analytical solutions to the lunar and planetary orbital motions, which implies the dependence of most of the long-period nutation terms on those fundamental arguments, denoted as

$$
\Theta_{i}=m_{i 1} l+m_{i 2} l^{\prime}+m_{i 3} F+m_{i 4} D+m_{i 5} \Omega .
$$

The subindex $i$ represent a 5 -tuple of integers $m_{i j} ; l, g$ and $h$ are the usual Delaunay variables of the Moon $(M) ; l^{\prime}, g^{\prime}$ and $h^{\prime}$ are those of the Sun $(S) ; F=l+g$, $D=l+g+h-l^{\prime}-g^{\prime}-h^{\prime}$, and $\Omega=h-\lambda$. Here $\lambda$ is the longitude of the node of the plane orthogonal to the angular momentum axis with respect to the ecliptic, reckoned in direct sense ${ }^{2}$.

At the first order of perturbation, the nutations in longitude, $\Delta \psi$, and obliquity, $\Delta \epsilon$, are given as sum of the so-called Poisson and Oppolzer terms, which provide, respectively, the perturbations due to action of the disturbing bodies, mainly Moon and Sun, on the angular momentum axis and the differential perturbations of the figure axis with respect to the former.

In the following expressions, originally developed in this way by Kinoshita (1977), the Poisson terms are those depending on functions $B_{i ; p}$ of the auxiliary angle $I_{0}$, while the Oppolzer terms contains functions $C_{i ; p}$. Recall that $I_{0}=-\epsilon_{A}(t)$ according to the perturbation method, although the strictly quasi-periodic nutation terms result from substituting $I_{0}=-\epsilon_{0}$, the constant term of $\epsilon_{A}$ in equations

\footnotetext{
2 Notice that we use the notation of Getino and Ferrándiz (1991) for the canonical variables, so that $\lambda$ corresponds to $h$ in Kinoshita's notation.
} 
(1). Namely, it turns out that (Kinoshita 1977)

$$
\begin{aligned}
\Delta \psi & =-\frac{1}{\sin I_{0}} \sum_{p=M, S} k_{p} \sum_{i \neq 0} \frac{1}{n_{i}} \frac{d B_{i ; p}}{d I}\left(I_{0}\right) \sin \Theta_{i}+\frac{1}{\sin I_{0}} \sum_{p=M, S} k_{p} \sum_{i} \sum_{\tau= \pm 1} \frac{\tau C_{i ; p}\left(I_{0}, \tau\right)}{n_{\mu}-\tau n_{i}} \sin \Theta_{i}, \\
\Delta \epsilon & =-\frac{1}{\sin I_{0}} \sum_{p=M, S} k_{p} \sum_{i \neq 0} \frac{1}{n_{i}} m_{i 5} B_{i ; p}\left(I_{0}\right) \cos \Theta_{i}+\sum_{p=M, S} k_{p} \sum_{i} \sum_{\tau= \pm 1} \frac{C_{i ; p}\left(I_{0}, \tau\right)}{n_{\mu}-\tau n_{i}} \cos \Theta_{i} .
\end{aligned}
$$

Here $n_{i}=\dot{\Theta}_{i}$ are the time derivatives of the nutation arguments $\Theta_{i}, n_{\mu}$ is the mean motion (or frequency) of the Andoyer variable $\mu$, close to the conventional mean value of the angular velocity of the Earth, $\omega_{E}$, and $k_{M}, k_{S}$ are the parameters introduced by Kinoshita (1977), defined by

$$
k_{p}=\frac{3 G m_{p}}{\omega_{E} a_{p}^{3}} H_{d}, \quad p=M, S \quad \text { (Moon, Sun) }
$$

which are proportional to the Earth's dynamical ellipticity $H_{d}$. The gravitational constant is denoted as $G, m_{p}$ stands for the mass of body $p$, and $a_{p}$ is the semimajor axis of its orbit.

The functions $B_{i ; p}$ and $C_{i ; p}$ depend on the orbital coefficients $A_{i}^{(j)}$ computed first by Kinoshita (1977), then by Kinoshita and Souchay (1990) and afterwards by Navarro (2001), from the analytical solutions ELP2000 and VSOP87 (ChaprontTouzé and Chapront 1983, Bretagnon 1988)

$$
\begin{aligned}
B_{i ; p}(I) & =-\frac{1}{6}\left(3 \cos ^{2} I-1\right) A_{i ; p}^{(0)}-\frac{1}{2} \sin 2 I A_{i ; p}^{(1)}-\frac{1}{4} \sin ^{2} I A_{i ; p}^{(2)} \\
C_{i ; p}(I, \tau) & =-\frac{1}{4} \sin 2 I A_{i ; p}^{(0)}+\frac{1}{2}(1+\tau \cos I)(-1+2 \tau \cos I) A_{i ; p}^{(1)}+\frac{1}{4} \tau \sin I(1+\tau \cos I) A_{i ; p}^{(2)}
\end{aligned}
$$

With respect to the precessional motion, the main component, $p_{A}^{\prime}$, of the general precession in longitude rate, $p_{A}$, is given by

$$
p_{A}^{\prime}=p_{M}^{\prime}+p_{S}^{\prime}=3 H_{d}\left[\left(\frac{m_{M}}{m_{M}+m_{E}}\right)\left(\frac{n_{M}^{2}}{\omega_{E}}\right) M_{0}+\left(\frac{m_{S}}{m_{S}+m_{E}+m_{M}}\right)\left(\frac{n_{S}^{2}}{\omega_{E}}\right) S_{0}\right] \cos \epsilon_{A}
$$


It is attributable to the first order secular solution (Kinoshita and Souchay, 1990), and the original notation there is kept: constants $M_{0}$ and $S_{0}$ are the orbital coefficients $A_{(0,0,0,0,0) ; M}^{(0)}$ and $A_{(0,0,0,0,0) ; S}^{(0)}$, respectively, $m_{B}$ represent the mass of each body $(B=E, M, S)$ and $n_{B}$ its relative mean motion. The observed value of the precession rate adopted in REN2000 was the one recommended by IAU at that time (the Lieske's constant, not the value updated in 2006). It was used to estimate $p_{A}^{\prime}$ and then to derive the value of $H_{d}$, and the resultant Kinoshita's constants $k_{M}$ and $k_{S}$, depending also of the system of astronomical constants in force those years (Lieske et al. 1977).

Souchay and Kinoshita (1996) already provided convenient expressions to update the value of the dynamical ellipticity as other parameters would vary, i.e.,

$$
\begin{aligned}
\delta H_{d}= & 6.4947 \times 10^{-7} \delta p_{A}+6.8812 \times 10^{-9} \delta \epsilon_{A}-4.4969 \times 10^{-3} \delta M_{0} \\
& -2.0708 \times 10^{-3} \delta S_{0}-0.17973 \delta \mu+1.03583 \times 10^{-3} \delta \rho,
\end{aligned}
$$

where here $\delta \mu$ and $\delta \rho$ represent the variations associated to the mass ratios between the Moon, the Earth, and the Sun.

The estimated value of $p_{A}^{\prime}$ (and thus $H_{d}$ ) changes not only along with variations of the observed precession rate, $p_{A}$, but with the changes of the precession of the ecliptic and various higher order contributions to precession, here denoted globally as $p_{S}$ (either by the updating of the values of some accounted effects or by the addition of new contributions), since $p_{A}^{\prime}+p_{S}=p_{A}$.

Non-rigid nutation theory MHB2000 is basically derived by convolving a certain nutation vector $\bar{\eta}_{R}\left(\sigma ; e_{R}\right)$, a complex linear combination of the nutations in longitude and obliquity, computed from the previous REN2000 solution after decomposing each periodic nutation in prograde and retrograde parts. After intro- 
ducing a generalized transfer function $T\left(\sigma ; e \mid e_{R}\right)$, the amplitude of each nutation term of the non-rigid Earth is given by $\bar{\eta}(\sigma ; e)=T\left(\sigma ; e \mid e_{R}\right) \bar{\eta}_{R}\left(\sigma ; e_{R}\right)$. In those expressions $e=(C-\bar{A}) / \bar{A}$ is a parameter close to the dynamical ellipticity $H_{d}$ and $\sigma$ is a forcing frequency relative to the rotating Earth, related to the time derivative of the nutation arguments.

The basic geophysical Earth model in MHB2000 has an elastic mantle, a fluid outer core (FOC), and a solid inner core (SIC), and its main features are gathered in a set of basic Earth parameters (BEP), some of them being fitted to observations. Effects of anelasticity and oceans were treated as corrections to some BEP, although e.g. they could have been alternatively incorporated into the integration of the dynamical equations of non-rigid Earth nutation by introducing complex elastic Lame parameters (Huang et al. 2001) and non-free stress boundary condition on the outer surface (Huang 2001). The model also considers electromagnetic and viscous couplings, or torques exerted by the fluid outer core on the mantle and on the inner core at the core-mantle boundary (CMB) and inner-core boundary (ICB), respectively.

The MHB2000 transfer function is given by a resonance formula depending on the normal mode frequencies $\sigma_{\alpha}$ of the basic Earth model and the relevant forcing frequencies $\sigma$

$$
T\left(\sigma ; e \mid e_{R}\right)=\frac{e_{R}-\sigma}{e_{R}+1} N_{0}\left[1+(1+\sigma)\left(\sum_{\alpha=1}^{4} \frac{N_{\alpha}}{\sigma-\sigma_{\alpha}}\right)\right], N_{0}=\frac{H_{d}}{H_{d R}}
$$

Let us remark that in the previous formula $H_{d R}$ stands for the dynamical ellipticity of REN2000, which is allowed to be given a new value $H_{d}$ in the computation of the MHB2000 transfer function for the sake of a better fitting of the theoretical to the VLBI observed amplitudes of a selected set of main nutation terms, which 
gave raise to the introduction of the additional parameter $N_{0}$. However, MHB2000 final nutation series were derived with a different transfer function, owing to a similar functional dependence but in which some of the geophysical parameters were changed from constants $\left(N_{\alpha}\right.$ and $\left.\sigma_{\alpha}\right)$ to linear functions of time $\left(Q_{\alpha}\right.$ and $\left.s_{\alpha}\right)$ in the diurnal band, also in order to improve the fitting to observations.

It may be not out of place to point out that nowadays MHB2000 model presents some shortcomings. For instance, it was early recognized (Getino and Ferrándiz 2000) that the transfer function treatment of second order effects, i.e., nutationnutation couplings proportional to $H_{d}^{2}$, is not correct, since this method is essentially linear - a fact also pointed out by Mathews et al. (2002). Yet another problem recently discussed is referred to the electromagnetic coupling at the CMB estimated in MHB2000 (Buffett et al. 2002). That mechanism was proposed to fill the dominant gap between the values of the period of the free core nutation (FCN) normal mode and the amplitude of retrograde annual nutation term either derived from theory or from observations, and stressed as one of the main advantages of that model over all other non-rigid Earth nutation models.

However, that coupling has been studied further and suspected broken by other works, e.g. Huang et al. (2011). In that investigation it is used the same model and values of the electromagnetic parameters crossing CMB as in Buffett et al. (2002). It was found that the contribution of this coupling to the retrograde annual nutation, and the change of the FCN period as well, is approximately one order of magnitude smaller than what is required, and also expected in Buffett et al. (2002), to fill the difference between the observations and the said theoretical models. Hence, there are other mechanisms that must play a more important 
role than previously considered in MHB2000 like, for example, viscous coupling, topographic coupling, etc. (see Huang et al. 2011 and references therein).

As a consequence of the foregoing description of the current IAU Earth rotation theory, the adoption of the precession model IAU2006 compels the adjustment of the IAU2000 nutation series to keep consistency within the set of successive IAU Resolutions (B1.6 adopted in 2000, B1 and B2 in 2006). In its turn, a right treatment of different non-rigid couplings of the nutation model, i.e., nutationnutation cross effects, entails the appearance of new contributions to the precession rates. Both issues lead to differences in the employed values of the dynamical ellipticity $H_{d}$ and are revised in the next sections.

\section{Changes of IAU2000 nutation theory from IAU2006 precession theory}

As explained in the last section the current IAU precession and nutation official models stem from separate dynamical theories. Needless to say that this is a valid approach, but current accuracy requirements demand a detailed analysis about the way in which these two parts are matched, in order to ensure the highest levels of consistency.

Next, we discuss the changes induced by the IAU2006 precession theory on the IAU2000 nutation theory. A comprehensive study of all the involved effects can be found in Escapa et al. (2014). Escapa et al. (2015) provide the theoretical background and technical details necessary to formulate those effects within the Hamiltonian framework. 
The adjustments considered in this work are due to the new features considered in the P03 precession model arising from the inclusion of the $J_{2}$ time rate, $\dot{J}_{2}$, in the modeling of the $J_{2}$ coefficient. Since the effects to be discussed here are at the micro-arcsecond ( $\mu$ as) level in the amplitude of each argument $\Theta_{i}$ of the nutation series, we consider a first order theory that accounts for the nutations of the angular momentum axis (Poisson terms), incorporating into the modeling the $J_{2}$ time rate. As it is well-known those nutations are common for one, two, and three-layer Earth models (Moritz and Mueller 1987).

The time evolution of the angular momentum axis is constructed by means of the Hamiltonian formalism. In the expression of the Hamiltonian, the Kinoshita's parameter $k_{p}$, which is proportional to the Earth's dynamical ellipticity, is replaced by a time-dependent function taking into account the $H_{d}$ time rate induced by the $J_{2}$ time rate. At first order, it has the form

$$
k_{p}=k_{p, 0}\left(1+t \frac{\dot{H}_{d}}{H_{d}}\right) \simeq k_{p, 0}\left(1+t \frac{\dot{J}_{2}}{J_{2}}\right)
$$

where $k_{p, 0}$ denotes now the time independent Kinoshita's parameter for Moon and Sun introduced in equations (3).

The computation of this effect (Escapa et al. 2014, 2015), following the perturbation equations, leads to the appearance of mixed secular terms (proportional to $t$, previously reported in Capitaine et al. 2005), and to in- and out-of-phase nutations. Namely, the following expressions to be summed to equations (3) are 
obtained

$$
\begin{aligned}
d_{j_{2}} \Delta \psi & =-\sum_{p=M, S}\left[t \frac{\dot{J}_{2}}{J_{2}} \frac{k_{p, 0}}{\sin I_{0}} \sum_{i \neq 0} \frac{1}{n_{i}} \frac{d B_{i ; p}}{d I}\left(I_{0}\right) \sin \Theta_{i}+\frac{\dot{J}_{2}}{J_{2}} \frac{k_{p, 0}}{\sin I_{0}} \sum_{i \neq 0} \frac{1}{n_{i}^{2}} \frac{d B_{i ; p}}{d I}\left(I_{0}\right) \cos \Theta_{i}\right], \\
d_{\dot{J}_{2}} \Delta \epsilon & =-\sum_{p=M, S}\left[t \frac{\dot{J}_{2}}{J_{2}} k_{p, 0} \sum_{i \neq 0} \frac{m_{i 5}}{n_{i}} \frac{B_{i ; p}\left(I_{0}\right)}{\sin I_{0}} \cos \Theta_{i}-\frac{\dot{J}_{2}}{J_{2}} k_{p, 0} \sum_{i \neq 0} \frac{m_{i 5}}{n_{i}^{2}} \frac{B_{i ; p}\left(I_{0}\right)}{\sin I_{0}} \sin \Theta_{i}\right] .
\end{aligned}
$$

To be consistent in the development of the theory, the inclusion of the $J_{2}$ time rate forces the consideration of the time rate of the orbital coefficients $A_{i}^{(0,1,2)}$ (Kinoshita 1977), due to the secular variation of Sun eccentricity (Escapa et al. $2014,2015)$. Analytically its treatment is similar to that of $J_{2}$ rate, providing also out-of-phase nutations and mixed secular terms with formulas similar to equations $(10)$.

Let us note that the mixed secular terms in equations (10) are proportional to $\dot{J}_{2} / J_{2}$, both in longitude and obliquity, in agreement with the result derived in Capitaine et al. (2005). Strictly speaking those expressions are only valid for the first order terms of the nutations, proportional to $H_{d}$, since some of the second order terms are proportional to $k_{p}^{2}$, i.e., to $H_{d}^{2}$, and the rescaling factor to be considered would be different from $\dot{J}_{2} / J_{2}$.

In contrast, the out-of-phase terms have not been considered previously and provide new relevant contributions at the $\mu$ as level given by

$$
d_{\dot{J}_{2}} \Delta \psi=-1.4 \cos \Omega, \quad d_{\dot{J}_{2}} \Delta \epsilon=-0.8 \sin \Omega
$$

In these expressions, and subsequent ones unless otherwise specified, the amplitudes are given in microarcseconds. 
Table 1 includes all the main related corrections arising from the $J_{2}$ time rate, comparing the mixed secular terms with the ones computed previously and currently included in IERS Conventions 2010.

Table 1 Corrections due to the inclusion of $J_{2}$ time rate

\begin{tabular}{|c|c|c|c|c|c|c|c|c|c|c|c|}
\hline \multirow{3}{*}{$\frac{1}{0}$} & \multirow[b]{3}{*}{0} & \multicolumn{3}{|c|}{$\Theta_{i}$} & \multirow{2}{*}{$\begin{array}{c}\text { Period } \\
\text { Days }\end{array}$} & \multicolumn{4}{|c|}{ Secular mixed terms $(\mu a s / c J)$} & \multicolumn{2}{|c|}{ Out-of-phase ( $\mu a s)$} \\
\hline & & $\mathrm{F}$ & $\mathrm{D}$ & $\Omega$ & & \multicolumn{2}{|c|}{$d \psi(t \times \sin )$} & \multicolumn{2}{|c|}{$d \epsilon(t \times \cos )$} & \multirow{2}{*}{$\frac{d \psi(\cos )}{-1.4^{2}}$} & \multirow{2}{*}{$\frac{d \epsilon(\sin )}{-0.8^{2}}$} \\
\hline & & 0 & 0 & 1 & -6798.36 & $47.8^{1}$ & $48.0^{2}$ & $-25.6^{1}$ & $-25.6^{2}$ & & \\
\hline 0 & 0 & 0 & 0 & 2 & -3399.18 & -0.6 & -0.6 & - & - & - & - \\
\hline 0 & 0 & 2 & -2 & 2 & 182.62 & 3.7 & 3.5 & -1.6 & -1.5 & - & - \\
\hline 0 & 0 & 2 & 0 & 2 & 13.66 & 0.6 & 0.6 & - & - & - & - \\
\hline
\end{tabular}

${ }^{1}$ Capitaine et al. (2005); ${ }^{2}$ Escapa et al. (2014)

The P03 precession model also introduced other changes in the numerical values of some precession parameters, which were different from Lieske et al. (1977). Specifically, the updating of the value of the obliquity $I_{0}=-\epsilon_{A}$ originates significative contributions, above the micro-arcsecond level, that should be envisaged as corrections to the IAU2000A nutation series in order to ensure its consistency with the IAU2006 precession. Corrections of this kind were first derived by Capitaine et al. (2005) who provide the terms

$$
d_{\epsilon_{A}} \Delta \psi=-8.1 \sin \Omega-0.6 \sin (2 F-2 D+2 \Omega)
$$

gathered in sec. 5.6.3 of the IERS Conventions 2010. A more detailed study falls out of the scope of this paper, which focuses on effects associated to the variations of the dynamical ellipticity. However, for the sake of completeness, let us say that the former corrections were completed by Escapa et al. (2014, 2015), the total 
effect being

$$
d_{\epsilon_{A}} \Delta \psi=(-15.6-8.1 t) \sin \Omega-0.6 \sin (2 F-2 D+2 \Omega), \quad d_{\epsilon_{A}} \Delta \varepsilon=0.8 \cos \Omega
$$

\section{Unaccounted contributions to the Earth's precession and effects on nutations}

With the precedent notations, the precession of the equator is made up of the main linear term $p_{A}^{\prime} t$ and various second order, smaller contributions. Let us recall that the expression "second order terms" is used in the literature to designate contributions of very different kinds. First, it means terms of various physical origins that produce linear perturbations of small magnitude. There are also second order terms in the sense of the perturbation methods, which are quadratic in the main perturbation parameter, which we can assimilate to $H_{d}$, so arising from the crossing of first order perturbations-asymptotically speaking. There were included in REN2000 for the rigid model, but a linear convolution with a transfer function is meaningless or doubtful since it does not fit well with the quadratic essence of those terms, as it was pointed out in Section 2.

Second order terms accounted in the precession theory have stayed unchanged since several years. A classic source is the paper by Williams (1994); Kinoshita and Souchay (1990) computed the full (quadratic) second order effects for the rigid earth, clarifying some differences of terminology and casting of terms with respect to Williams in their 1997 paper (Souchay and Kinoshita 1997). Those former contributions are the same shown by Capitaine et al. (2003) - apart from some slight adaptations. 
However, a few, significant, new contributions have been found in the last years. The most remarkable are due to: (1) non-rigid quadratic second order mathematical solution (Ferrándiz et al. 2004, 2007); (2) effects of the mantle anelasticity (Lambert and Mathews 2006 coupled to oceans, as well as Ferrándiz et al. 2012, Baenas 2014, uncoupled). Following the analytical procedure described above, the Hamiltonian approach provided a systematic procedure to compute both kinds of terms, which guarantees the consistency of the results; the method proceeds by adding the new relevant terms to the basic Hamiltonian and then applying the same perturbation method to the extended Hamiltonian.

\subsection{Unaccounted effects on non-rigid Earth precession}

After P03 precession model was adopted, several research works have identified non-negligible effects on non-rigid Earth precession. Their addition to the existing models is equivalent to a suitable change of the determined (or observed) value of precession, producing thus an equivalent change of the value of the main coefficient $p_{A}^{\prime}$. Therefore, the derived parameter $H_{d}$ varies accordingly and the whole set of nutations suffers and indirect effect along with that change. Notice that, the quadratic rigid second order effects on precession being part of REN2000, they must be subtracted from those computed from a more realistic non-rigid Earth model to derive the effective variations of them. Table 2 shows examples of such kind of new contributions, extracted from Ferrándiz et al. (2007) and Baenas (2014), considering different elastic responses of the Earth's mantle. 
Table 2 Corrections due to unaccounted effects on precession

\begin{tabular}{ccccc}
\hline Effect (diff. WRT rigid model) & $\Delta$ Longitude & $\Delta$ Ellipticiy & $\Delta$ Obliquity \\
\hline Earth model & Perturb. order & $d p_{\psi}(\mathrm{mas} / \mathrm{cy})$ & $\delta H_{d}(\mathrm{ppm})$ & $d p_{\epsilon}(\mathrm{mas} / \mathrm{cy})$ \\
\hline Poincaré & 2 & -23.1578 & 4.8 & - \\
Fluid core + Elastic mantle & 2 & -12.5673 & 2.5 & - \\
Mantle anelasticity & 1 & 6.0059 & -1.2 & 0.7748 \\
\hline
\end{tabular}

4.2 Indirect effects on nutation

Indirect effects on nutation are those resulting from the changes in the value of the main precession parameter $H_{d}$, as discussed above. Their derivation is simple, since it suffices to compute the linear approximation by multiplying the nutation amplitudes by the factor $\left(H_{d}+\delta H_{d}\right) / H_{d}$, either in the fully non-rigid Hamiltonian approach or the rigid Hamiltonian + non-rigid transfer function approaches. The main terms arising from $\left(\delta H_{d}\right) / H_{d}$ are displayed in Table 3. Note that some of them reach tens of $\mu$ as, well above the accuracy objective pursued nowadays.

Table 3 Corrections due to indirect effects on nutation

\begin{tabular}{|c|c|c|c|c|c|c|c|c|c|}
\hline \multicolumn{5}{|c|}{$\Theta_{i}$} & \multirow{2}{*}{$\frac{\text { Period }}{\text { (days) }}$} & \multicolumn{2}{|c|}{ Poincaré model ( $\mu$ as) } & \multicolumn{2}{|c|}{ Fluid core + Elasticity ( $\mu$ as) } \\
\hline$l$ & $l^{\prime}$ & $F$ & $D$ & $\Omega$ & & $\Delta \psi(\sin )$ & $\Delta \epsilon(\cos )$ & $\Delta \psi(\sin )$ & $\Delta \epsilon(\cos )$ \\
\hline 0 & 0 & 0 & 0 & 1 & -6798.36 & -82.59 & 44.19 & -44.64 & 23.93 \\
\hline 0 & 0 & 0 & 0 & 2 & -3399.18 & 1.00 & -0.43 & - & - \\
\hline 0 & 0 & 2 & -2 & 2 & 182.62 & -6.32 & 2.75 & -3.42 & 1.49 \\
\hline 0 & 0 & 2 & 0 & 2 & 13.66 & -1.09 & 0.47 & - & - \\
\hline
\end{tabular}




\section{Conclusions}

Current research on Earth rotation modeling, encouraged by the IAU/IAG Joint Working Group on Theory of Earth Rotation, has to face the problem of obtaining very demanding accuracy goals, set as $30 \mu a s$ and $3 \mu a s / y$ in orientation and velocity, respectively, as shown, e.g., in Ferrándiz and Gross (2014a, 2014b). This is the same very stringent level of accuracy first established by the GGOS of the IAG, see, e.g., Plag and Pearlman (2009) for a comprehensive description of the geodetical problems and the expected societal benefits which led to such a high accuracy requirements, including the geodetical monitoring of the global change. The consistency of the current precession and nutation theories (derived successively in time as a set of three main pieces, based on mathematically different approaches) is an obvious requirement, although it is not fully achieved at such level of accuracy, but additional corrections must be added to the existing ones. Although only few nutation terms require not negligible corrections due to the differences of the dynamical ellipticity values used in the IAU2000 and IAU2006 models, as shown in this paper, those corrections cannot be ignored any more, since they are needed to ensure the consistency of the current precession-nutation models.

\footnotetext{
Acknowledgements The authors thank the editor M. Charco, C.L. Huang and a second anonymous referee for their valuable advice, which helped to improve the manuscript. This work has been partially supported by the Spanish government under grant AYA2010-22039C02-02 from Ministerio de Economía y Competitividad (MINECO) and Generalitat Valenciana project GV/2014/072.
} 


\section{References}

[2014] Baenas, T. (2014), Contribuciones al estudio analítico del movimiento de rotación de una Tierra deformable (in Spanish), PhD thesis, University of Alicante.

[2012] Böhm, S., Brzezinski, A., Schuh, H. (2012), Complex demodulation in VLBI estimation of high frequency Earth rotation components, J. Geodyn., 62, 56-58.

[1988] Bretagnon, P. (1988), Planetary theories in rectangular and spherical variables. VSOP87 solution, Astron. Astrophys. 202, 304-315.

[2002] Buffet, B.A., Mathews, P.M., Herring, T.A. (2002), Modeling of nutation and precession: Effects of electromagnetic coupling, J. Geophys. Research 107, No. B4, 2070.

[2003] Capitaine, N., Wallace, P.T., Chapront, J. (2003), Expressions for IAU2000 precession quantities, Astron. Astrophys. 412, 567-586.

[2005] Capitaine, N., Wallace, P.T., Chapront, J. (2005), Improvement of the IAU2000 precession model, Astron. Astrophys. 432, 355-367.

[1983] Chapront-Touzé, M., Chapront, J. (1983), The lunar ephemeris ELP-2000, Astron. Astrophys. $124,50-62$.

[2014] Escapa, A., Getino, J., Ferrándiz, J., Baenas, T. (2014), On the changes of the IAU2000 nutation theory stemming from IAU 2006 precession theory, In: Capitaine, N. (ed) Proceedings of the Journées 2013 Systèmes de Référence Spatio-Temporels, Observatoire de Paris, $148-151$.

[2015] Escapa, A., Getino, J., Ferrándiz, J. M., Baenas, T. (2015), Dynamical matching between IAU2000 nutation theory and IAU 2006 precession theory (in preparation)

[1991] Getino, J., Ferrándiz, J. M. (1991), A Hamiltonian Theory for an Elastic Earth: Elastic Energy of Deformation, Celes. Mech. 51, 17-34.

[2000] Getino, J., Ferrándiz, J. M. (2000), Towards models and constants for submicroarcsecond astrometry, In: Johnston, K. J., McCarthy, D. D., Luzum, B. J., Kaplan, G. H. (eds) Proceedings of IAU Colloquium 180, U. S. Naval Observatory, Washington, DC, USA, 236-241.

[2014a] Ferrándiz, J. M. and Gross, R.S. (2014), The New IAU/IAG Joint Working Group on Theory of Earth Rotation, IAG Symp 143 (to appear). 
[2014b] Ferrándiz, J. M. and Gross, R.S. (2014), The goal of the IAU/IAG Joint Working Group on the Theory of Earth rotation, In: Capitaine, N. (ed) Proceedings of the Journées 2013 Systèmes de Référence Spatio-Temporels, Observatoire de Paris, 139-143.

[2004] Ferrándiz, J.M., Navarro, J.F., Escapa, A., Getino, J. (2004), Precession of the nonrigid Earth: effect of the fluid outer core, Astron. J. 128, 1407-1411.

[2007] Ferrándiz, J.M., Navarro, J.F., Escapa, A., Getino, J., Baenas, T. (2007), Influence of the mantle elasticity on the precessional motion of a two-layer Earth model, In: Lemaittre, A. (ed) The rotation of celestial bodies, Press. Universitaires de Namur, 9-14.

[2012] Ferrándiz, J.M., Baenas, T., Escapa, A. (2012), Effect of the potential due to lunisolar deformations on the Earth precession. Geophysical Research Abstracts, 14, EGU2012-6175.

[2003] Fukushima, T. (2003), A new precession formula, Astron. J. 126, 494-534.

[2015a] Heinkelmann, R., Belda-Palazón, S., Ferrándiz, J.M., Schuh, H. (2015) The consistency of the current conventional celestial and terrestrial reference frames and the conventional EOP series, In: Capitaine, N., Malkin, Z. (eds) Proceedings of the Journées 2014 Systèmes de Référence Spatio-Temporels, 224-225

[2015b] Heinkelmann, R., Belda-Palazón, S., Ferrándiz, J.M., Schuh, H. (2015) How consistent are the current conventional celestial and terrestrial reference frames and the conventional Earth orientation parameters?, IAG Symp (Proc. of Reference Frames for Applications in Geosciences, REFAG2014, accepted 2015)

[1967] Heiskanen, W. A., Moritz, H., Physical Geodesy ( W. H. Freeman \& Co. Ltd. 1967).

[2006] Hilton, J.L., Capitaine, N., Chapront, J., Ferrándiz, J.M., Fienga, A., Fukushima, T., Getino, J., Mathews, P., Simon, J.L., Soffel, M., Vondrak, J., Wallace, P., Williams, J. ( 2006), Report of the International Astronomical Union Division I Working Group on Precession and the Ecliptic, Celest. Mech. Dyn. Astron. 94, 351-367. doi:10.1007/s10569006-0001-2.

[1966] Hori, G. I. (1966), Theory of general perturbations with unspecified canonical variables, Publ. Astron. Soc. Jpn. 18, 287-296.

[2001] Huang C. L. (2001), The scalar boundary conditions for the motion of the elastic Earth to second order in ellipticity, Earth, Moon, and Planets, 84, 125-141.

[2001] Huang, C. L., Jin, W. J., Liao, X. H. (2001), A new nutation model of a non-rigid Earth with ocean and atmosphere, Geophys. J. Int., 146, 126-133. 
[2011] Huang, C. L., Dehant V., Liao X. H., Van Hoolst T., Rochester M.G. (2011), On the coupling between magnetic field and nutation in a numerical integration approach, J. Geophys. Res., 116, No. B03403.

[2014] Huang, C. L., Liu, C. J., Liu, Y. (2014), A generalized theory of the figure of the Earth: application to the moment of inertia and global dynamical flattening, In: Capitaine, N. (ed) Proceedings of the Journées 2013 Systèmes de Référence Spatio-Temporels, Observatoire de Paris, 156-159.

[1975] Kinoshita, H. (1975), Formulas for precession, Smithsonian Astrophys. Obs. Special Report, No. 364 .

[1977] Kinoshita, H. (1977), Theory of the rotation of the rigid Earth, Celest. Mech. 15, 277-326.

[1990] Kinoshita, H., Souchay, J. (1990), The theory of the nutation for the rigid-Earth model at the second order, Celest. Mech. 48, 187-265.

[2006] Lambert, S.B., Mathews, P.M. (2006), Second-order torque on the tidal redistribution and the Earth's rotation, Astron. Astrophys. 453, 363-369.

[1977] Lieske J., Lederle T., Fricke W., Morando B. (1977), Expression for the precession quantities based upon the IAU (1976) system of astronomical constants, Astron. Astrophys. $58,1-16$.

[2002] Mathews, P.M., Herring, T.A., Buffet, B.A. (2002), Modeling of nutation and precession: New nutation series for nonrigid Earth and insights into the Earth's interior, J. Geophys. Research 107, No. B4, 2068.

[1987] Moritz, H., Mueller, I., Earth Rotation (Frederic Ungar 1987).

[2001] Navarro, J.F. (2001), Teoría analítica de la rotación de la Tierra rígida mediante manipulación simbólica específica (in Spanish), PhD thesis, University of Alicante.

[2010] Nilsson, T., Böhm, J., Schuh, H.( 2010), Sub-diurnal earth rotation variations observed by VLBI, Artificial Satellites, Vol. 45, No. 2. doi: 10.2478/v10018-010-0005-8.

[2010] Petit, G. and Luzum, B.,(eds.) IERS Conventions 2010, IERS Technical Note 36, Verlag des Bundesamtes für Kartographie und Geodäsie, Frankfurt am Main.

[2009] Plag, H.-P., Gross, R., Rothacher, M. (2009), Global Geodetic Observing System for Geohazards and Global Change. Geosciences, BRGM's journal for a sustainable Earth, 9, 96-103. 
[2009] Plag, H. P. and Pearlman, M. (eds) (2009), Global Geodetic Observing System: Meeting the Requirements of a Global Society on a Changing Planet in 2020, Springer-Verlag, Berlin-Heidelberg.

[1996] Souchay, J. and Kinoshita, H. (1996), Corrections and new developments in rigid earth nutation theory. I. Lunisolar influence including indirect planetary effects, Astron. Astrophys. $312,1017-1030$.

[1997] Souchay, J. and Kinoshita, H. (1997), Corrections and new developments in rigid-Earth nutation theory. II. Influence of second-order geopotential and direct planetary effect, Astron. Astrophys. 318, 639-652.

[1999] Souchay, J., B. Losley, H. Kinoshita, Folgueira, M. (1999), Corrections and new developments in rigid earth nutation theory. III. Final tables "REN-2000" including crossednutation and spin-orbit coupling effects. Astron. Astrophys. Suppl. Ser., 135, 111-131.

[1994] Williams J.G. (1994), Contributions to the Earth's obliquity rate, precession, and nutation. Astron. J. 108, 2, 711-724. 